\title{
The circumbinary dusty disk around the hydrogen-deficient binary star $v$ Sagittarii
}

\author{
M. Netolický ${ }^{1,2}$, D. Bonneau ${ }^{3}$, O. Chesneau ${ }^{3}$, P. Harmanec ${ }^{4}$, P. Koubský ${ }^{1}$, D. Mourard ${ }^{3}$, and P. Stee ${ }^{3}$ \\ 1 Astronomical Institute of the Academy of Sciences, 25165 Ondřejov, Czech Republic \\ e-mail: netol@physics.muni.cz \\ 2 Masaryk University, Faculty of Science, Department of Theoretical Physics and Astrophysics, Kotlářská 2, 61137 Brno, \\ Czech Republic \\ 3 UMR 6525 H. Fizeau, Univ. Nice Sophia Antipolis, CNRS, Observatoire de la Côte d'Azur, Av. Copernic, 06130 Grasse, France \\ 4 Astronomical Institute of the Charles University, Faculty of Mathematics and Physics, V Holešovičkách 2, 18000 Praha 8, \\ Czech Republic
}

Recieved 20 October 2008 / Accepted 2 December 2008

\section{ABSTRACT}

\begin{abstract}
Aims. The aim of this paper is to determine the properties of the dusty environment of the hydrogen-deficient binary system $v$ Sgr, whose binary properties and other characteristics are poorly known.

Methods. We obtained the first mid-IR interferometric observations of $v$ Sgr using the instrument MIDI of the VLTI used with different pairs of $1.8 \mathrm{~m}$ and $8 \mathrm{~m}$ telescopes. The calibrated visibilities, the $N$ band spectrum, and the SED were compared with disk models computed with the MC3D code to determine the geometry and chemical composition of the envelope.

Results. $v$ Sgr is unresolved with an $8 \mathrm{~m}$ telescope at $8.7 \mu \mathrm{m}$. We propose a disk model that agrees with the measured visibilities and the SED, consisting of a geometrically thin disk with an inner radius $R_{\text {in }}=6.0_{-1.5}^{+0.5} \mathrm{AU}$ and a scale height $h_{100}=3.5_{-1.5}^{+2.0} \mathrm{AU}$. The chemical composition of the dust is approximately $60 \%$ of carbon dust and $40 \%$ of silicate dust, as a consequence of several episodes of mass transfers, whose chemistry was imprinted in the dust composition. We also constrain the inclination of the disk $i=50_{-20^{\circ}}^{\circ}$ and its orientation position angle $\mathrm{PA}=80_{-5^{\circ}}^{\circ}$.

Conclusions. The mid-infrared interferometric observations of the binary star $v$ Sgr allowed us to constrain the geometry of the circumbinary dusty envelope. By defining the inclination and PA of the system with better accuracy than before, these observations restrict the parameter space for the orbital parameters and thus the nature of the stars orbiting in this system.
\end{abstract}

Key words. stars: emission-line, Be - stars: binaries: close - stars: circumstellar matter - techniques: interferometric stars: individual: $v$ Sagittarii

\section{Introduction}

Close binaries are complex objects from both the observational and modeling perspectives. Because of the interaction between the components, physical processes like mutual reflection, tidal interaction, mass loss, and mass transfer have to be taken into account when investigating the structure, physical conditions, and evolution of these objects. The interest of the observations of circumstellar matter in close binary systems was presented by Kuiper (1941) and the first review on the subject was published by Batten (1970).

Both spectroscopic and photometric observations of interacting binaries show that the structure of circumstellar environment is very complicated in many cases. As an example, an accretion disk, gaseous streams, jets, and scattering envelopes were found and studied in the well-known interacting binary $\beta$ Lyrae (Harmanec et al. 1996; Harmanec 2002; Hoffman et al. 1998; Ak et al. 2007; Schmitt 2008; Zhao et al. 2008). With the exception of eclipsing binaries, presence of the circumstellar structures and circumbinary envelopes is mainly deduced from the often ambiguous transformation from the velocity into Cartesian space. From this point of view, interferometry or, even more,

\footnotetext{
* Based on observations made with the Very Large Telescope Interferometer at Paranal Observatory under program 079.D-0115.

$\star \star$ Visibility data are only available in electronic form at the CDS website.
}

spectro-interferometry can bring a new insight into these complicated systems, permitting, at least, a limited but direct imaging of the circumstellar structures.

The system $v$ Sgr (HD 181615, HR 7342, HIP 95176) with an A type low-mass supergiant, is the brightest member of the type of extremely hydrogen-deficient binaries stars (HdB stars). The HdB stars are a rather rare class of evolved binary systems that are in a second phase of mass transfer where the primary has ended the core helium-burning phase (Schoenberner \& Drilling 1983). The parallax of $v$ Sgr determined by Hipparcos (1.95 \pm 0.72 mas, Perryman et al. 1997) has been recently revised to $1.68 \pm 0.23$ mas (van Leeuwen 2007) giving a distance of $d=$ $595_{-72}^{+94}$ pc. Koubský et al. (2006) constrained the semi-major axis of the binary ranging from $221 R_{\odot}$ to $415 R_{\odot}$. This corresponds to the angular size ranging from 2.0 mas to 3.8 mas when adopting the parallax given by Perryman et al. (1997) and to angular size ranging from 1.7 mas to 3.2 mas when adopting the parallax given by van Leeuwen (2007).

Extensive studies of $v$ Sgr with spectrometric and photometric techniques provide a basic picture of the object, but also many open questions. The goal of the interferometric observations is to improve our knowledge about this puzzling object.

The system $v$ Sgr has long been known as a single-lined spectroscopic binary $(P=137.9 \mathrm{~d})$. Its radial velocity variations were discovered by Campbell (1899) and the first orbital elements computed by Wilson (1913). The secondary 
orbit was determined by the cross-correlation technique applied to the IUE spectra (Dudley \& Jeffery 1990). The secondary ("invisible") component seems to be more massive $(q=1.59)$, while the luminosity ratio of "visible" to "invisible" seems to be about 100. However, the detection of the secondary lines is uncertain, given the low SNR of the IUE spectra so it requires independent verification. Numerous photometric observations by several authors did not provide any evidence of binary eclipses but are indicative of variations on a time scale of about 20 (or 40) days as mentioned in Frame et al. (1995) (see references in Koubský et al. 2006).

The majority of the spectroscopic studies concerning $v$ Sgr was devoted to the primary spectrum, which combines the absorption lines of low and high degrees of ionization and the $\mathrm{H} \alpha$ emission. Hack \& Pasinetti (1963) demonstrated that the chemical composition of the primary star is a peculiar one strong hydrogen deficiency and overabundance of $\mathrm{C}$ and $\mathrm{N}$ and the evolutionary scenarios for $v$ Sgr as an HdB star were presented by Schoenberner \& Drilling (1983) and by Eggleton (2002). The most compelling evidence of circumstellar matter in $v$ Sgr comes from the complex $\mathrm{H} \alpha$ absorption/emission profile and the large infrared excess. It is now well documented that the overall shape of the $\mathrm{H} \alpha$ profile alternates between two distinct appearances, on a time scale that remains undefined, but that is definitely longer than several times the binary orbital period:

1. Stage A: a quiet phase. It is characterized by an almost stationary emission peak $\left(\sim+10 \mathrm{~km} \mathrm{~s}^{-1}\right)$ with a redward extended emission wing $\left(\sim+10\right.$ to $\left.+500 \mathrm{~km} \mathrm{~s}^{-1}\right)$ and no blueshifted absorption. The variations observed do not seem to follow the orbital motion of the system. A possible explanation suggests that this stage is associated with a non-uniform shell surrounding the system (Frame et al. 1995).

2. Stage B: an active phase. It is characterized by the $\mathrm{H} \alpha$ profile composed of an emission line peak without a redward extended wing and a blue-shifted absorption component $\left(\sim-300 \mathrm{~km} \mathrm{~s}^{-1}\right)$. The emission component is almost stationary and the radial velocity of the absorption component changes with the orbital period, but the changes cannot be easily described. The active phase has been recorded by various observers several times since 1949 and has been observed since 2005.

Nariai (1967) proposed that the displaced $\mathrm{H} \alpha$ absorption is formed in a supersonic flow generated as the gas is transferred from the primary via the L1 point and that it adopts a form of a cone directed toward the secondary and partly escapes from the system in the form of an outflowing spiral arm encircling the whole binary. The proposed geometry is reminiscent of the pinwheel nebula discovered much later by Tuthill et al. (1999), but within the different physical context of mass-exchange between the component, and not of a wind-wind collision in a Wolf-Rayet/OB binary. Koubský et al. (2006) show that the blueshifted absorption in the $\mathrm{H} \alpha$ is visible throughout the whole orbital cycle (though with strongly varying strength), and they derived the radial-velocity curve that seems to challenge the Nariais model. These authors therefore tentatively suggest an alternative model: $v$ Sgr might be a non-eclipsing analog of the $\beta$ Lyr system, provided that the peculiar spectrum of the primary would come from the inner rim of an inclined disk, while the blue-shifted absorption would originate from the slowly precessing bipolar jets.

The circumbinary material is also shown by the strong infrared excess of $v$ Sgr and, in particular, by the prominent silicate dust signature at $9.7 \mu \mathrm{m}$ (Gehrz et al. 1974). The large infrared excess causes an apparent increase in the angular diameter with the wavelength when estimated via the infraredflux method of Shallis \& Blackwell (1979). From the analysis of IRAS photometric measurements and low-resolution spectra, Walker (1985) constrained the dust extension based on a spherical dust shell model with the following parameters: $T_{\text {dust }} \sim$ $1000 \mathrm{~K}, r_{\text {dust }} / R_{\text {star }}>40$. This author also noted that the silicate feature in the $v$ Sgr spectrum is rather puzzling since this star is deficient in oxygen (Hack \& Pasinetti 1963). This situation is clearly reminiscent of the double-chemistry dust observed in some post-AGB stars, for which a long-lived dusty disk traps and stores part of the mass ejected during the early phases of mass transfer (Waters et al. 1998).

Our paper reports on MIDI/VLTI observations of the dusty environment of $v$ Sgr and is organized as follow. In Sect. 2 we present the VLTI interferometric observations and results of the data processing leading to the calibrated visibilities. Section 3 presents the best model obtained for the dusty envelope of $v$ Sgr using interferometric and photometric data as constraints for the Monte-Carlo radiative transfer code MC3D. The results are the discussed in Sect. 4. Finally, in Sect. 5 the results are summarized and some conclusions drawn from the first midinfrared interferometric measurements of the circumbinary envelope of $v$ Sgr.

\section{Observation and data analysis}

\subsection{Description of the observed data}

The observations were carried out with the instrument MIDI of the Very Large Telescope Interferometer (VLTI) in operation at the ESO Paranal Observatory (Leinert et al. 2003), using the $1.8 \mathrm{~m}$ Auxiliary Telescopes (ATs) and $8 \mathrm{~m}$ Unit Telescopes (UTs). The source $v$ Sgr was observed from April to August 2007 and in May 2008 with baselines ranging from $20 \mathrm{~m}$ up to $125 \mathrm{~m}$. The journal of the observations is given in Table 1.

The observations were performed with the PRISM dispersive element giving a spectral resolution of $R \sim 30$ in the $N$-band $(8-13 \mu \mathrm{m})$. Twelve measurements of the object were used for the data processing. The data recorded with the UTs were in SCI_PHOT mode (i.e. with simultaneous photometry), and the ATs ones in HIGH_SENS mode (with photometry recorded subsequently, leading to an increase the errors). Further reference for one particular observation will be given within the number of observation listed in the first column of this table enclosed by brackets.

Due to a lower sensitivity and a lower ratio between the extension in pixel between the ATs beam and field-of-view (FOV), it is more difficult to extract any accurate photometry. However, a careful inspection of the acquisition images recorded with the UTs shows that, even for a $8 \mathrm{~m}$ telescope, the dusty environment of $v \mathrm{Sgr}$ is unresolved. Indeed, the same level of flux was obtained for the $N$ band spectrum of ATs and UTs, but we only show the best spectrum recorded in Fig. 3 .

The images were recorded using the MIDI star acquisition modes with the $8.7 \mu \mathrm{m}$ filter. The acquisition mode is the first template used after the pointing to test if the target is within the MIDI FOV (diameter of about $3^{\prime \prime}$ for UTs, and about 6" for ATs) and to perform a fine pointing. The chopping mode $(f=2 \mathrm{~Hz}$, angle $-90^{\circ}$ ) is used to visualize the star, which is not perfectly centered in the first image, and centered in a second step. The number of frames recorded per image is generally about 2000 
Table 1. Journal of observations for $v$ Sgr. The 7th column gives the information, how many visibility measurements were obtained for each observation. The error in 8th column is the adopted uncertainty of visibility in percent derived from scatter of calibrated visibilities. In the cases where only one calibrator can be used, we estimated the error from calibrated visibilities also using suspicious calibrators, which were not taken into account for computing the mean visibility.

\begin{tabular}{|c|c|c|c|c|c|c|c|}
\hline \multirow[t]{2}{*}{ No. } & \multirow[t]{2}{*}{ Date } & \multirow[t]{2}{*}{ Base } & \multicolumn{2}{|c|}{ Projected baseline } & \multirow{2}{*}{$\begin{array}{l}\text { Calibrators } \\
\text { HD number }\end{array}$} & \multirow[t]{2}{*}{ Nbr } & \multirow{2}{*}{$\begin{array}{l}\text { Err } \\
{[\%]}\end{array}$} \\
\hline & & & $\begin{array}{c}\text { length } \\
{[\mathrm{m}]}\end{array}$ & $\begin{array}{c}\text { PA } \\
{[\operatorname{deg}]}\end{array}$ & & & \\
\hline (1) & 2007-06-22T09 & G0-H0 & 20.3 & 82.1 & $124897 ; 167618 ; 168454$ & 1 & 10 \\
\hline (2) & 2007-06-22T02 & G0-H0 & 20.8 & 36.2 & $124897 ; 167618 ; 168454$ & 1 & 10 \\
\hline (3) & 2007-07-04T07 & G0-H0 & 26.6 & 79.7 & 168454 & 1 & 20 \\
\hline (4) & 2007-06-22T06 & G0-H0 & 31.8 & 73.7 & $124897 ; 167618 ; 168454$ & 1 & 10 \\
\hline (5) & 2007-05-17T05 & D0-H0 & 45.6 & 44.4 & $168454 ; 177716$ & 1 & 15 \\
\hline (6) & 2007-04-24T09 & D0-H0 & 63.2 & 69.1 & 167618 & 3 & 10 \\
\hline (7) & 2007-05-08T09 & D0-G1 & 69.7 & 138.5 & 167618 & 2 & 10 \\
\hline (8) & 2007-04-10T07 & U1-U4 & 96.0 & 26.5 & 169916 & 1 & 20 \\
\hline (9) & 2008-05-17T10 & U1-U4 & 123.5 & 66.6 & 142804,175775 & 1 & 5 \\
\hline
\end{tabular}

and the exposure time is by default $4 \mathrm{~ms}$ in order to avoid background saturation.

In order to calibrate the visibilities of the science target, we used observations of calibrating stars obtained the same night with the same instrumental setup. These stars were selected from the MIDI calibrator selected using the SearchCal tool available at $\mathrm{JMMC}^{1}$ and it was supposed that their spatial brightness distribution can be described in the mid-IR with a uniform disk of any given diameter. The overview of the calibrators and their properties is given in Table 2. All of them are standard MIDI calibrators and the angular diameters were taken from the MIDI calibrator database. Fluxes listed in the table are the IRAS $12 \mu$ m fluxes. The last column shows which calibrators were used for UTs or ATs observations.

\subsection{Data reduction}

The data obtained with the MIDI instrument were reduced using IDL-based MIA+EWS software. A description of the data reduction steps can be found in Leinert et al. (2004). We followed the standard reduction process. To increase the accuracy of the measurements, we spectrally binned the data obtained with ATs with boxes of 5 pixels.

During the data reduction we tried to use the maximum available set of the calibrators, but some calibrators were observed during unfavorable conditions. They gave inconsistent results and were excluded from any more reduction. For the observation (8) with UTs, one calibrator was rejected because of the bad weather conditions. For the observations made with the ATs, the HIGH_SENS mode used for the data acquisition is very sensitive to the fluctuations in the transparency and seeing, sometimes leading to visibilities $>1$. For this reason, one calibrator was rejected for the observations $(1,2,4,6,7)$ and 3 calibrators were rejected for observation (3). To increase accuracy we used the binning parameter of 5 .

As seen in Fig. 1, the dataset offers quite reasonable coverage of the uv space, but there is only 1 measurement (7) in position angle range from $90^{\circ}-180^{\circ}$. This can be the main source of uncertainty in determining of the inclination angle.

\subsection{Simple geometric model}

The source visibility curves are best-fitted with 2D elliptical Gaussian as a first-order model approximation of our data. This

1 http://www $\cdot$ mariotti.fr/
Table 2. Main parameters of the calibrators.

\begin{tabular}{ccccc}
\hline \hline $\begin{array}{c}\text { HD } \\
\text { number }\end{array}$ & $\begin{array}{c}\text { Spectral } \\
\text { type }\end{array}$ & $\begin{array}{c}\text { Flux } \\
{[\mathrm{Jy}]}\end{array}$ & $\begin{array}{c}\text { Ang. diam. } \\
{[\mathrm{mas}]}\end{array}$ & Telescope \\
\hline 124897 & K1.5III & 547 & $19.26 \pm 0.01$ & AT \\
142804 & M1III & 8.1 & $2.77 \pm 0.61$ & UT \\
167618 & M3.5III & 149 & $11.33 \pm 0.04$ & AT \\
168454 & K3IIIa & 43.7 & $5.78 \pm 0.15$ & AT \\
169916 & K1IIIb & 21.6 & $3.75 \pm 0.04$ & UT \\
175775 & G9II/III & 13.7 & $3.26 \pm 0.23$ & UT \\
177716 & K1IIIb & 18.0 & $3.72 \pm 0.07$ & AT \\
\hline
\end{tabular}

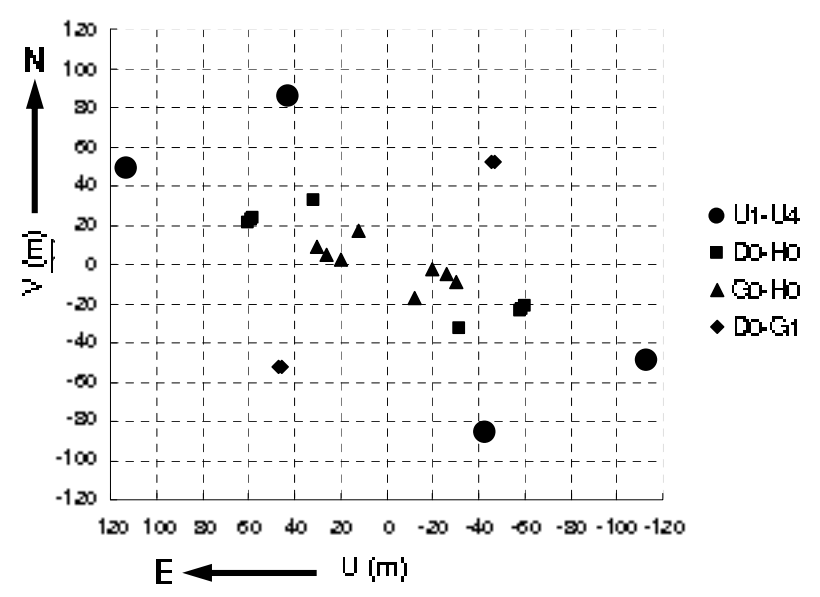

Fig. 1. The uv coverage over the time of observations of the VLTI baselines using UTs (U1-U4) and ATs (D0-H0, G0-H0, D0-G1).

is not surprising for such a dusty source, and we also do not expect the stars to be detected and resolved in the mid-IR. Even though the geometry of the dusty envelope is probably complex, such fits can provide a basic picture of the dusty envelope, i.e. the direction of the major axis and the inclination of the system. All visibility spectra were split into $1 \mu \mathrm{m}$ bins, and we fitted the average visibilities of each bin using independent models. In the $N$-band $(\lambda=10 \mu \mathrm{m})$, we found the average FWHM along the major axis $a=(20.0 \pm 1.4)$ mas and FWHM along the minor axis $b=(14.2 \pm 2.9)$ mas. These results provide an estimate of the inclination, $i=45_{-14^{\circ}}^{\circ}$, derived from the ratio of the minor and the major axes, assuming an intrinsic circular structure. The fits give also a consistent estimate of the orientation of the dusty envelope of $\mathrm{PA}=76^{\circ} \pm 27^{\circ}$, roughly perpendicular to the polarization of the source (Yudin 2001). 


\section{Radiative transfer modeling}

The interferometric mid-IR calibrated visibilities have been combined with the spectral energy distribution (SED) to constrain the physical parameters of the dusty envelope of $v$ Sgr using a model computed with the radiative transfer code MC3D.

\subsection{About the reddening of $v$ Sgr}

For $v$ Sgr, only moderate values of $E(B-V)$ ranging from 0.1 to 0.3 are found in the literature. From the analysis of the ultraviolet spectrum observed by the $\mathrm{S} 2 / 68$ experiment of the TD1 satellite, Duvignau et al. (1979) indicated a best $E(B-V)$ of 0.20 , always less than 0.30 . Using low-resolution IUE spectrum, Schoenberner \& Drilling (1984) found a value of 0.12 . From the UV photometric observations of $v$ Sgr by ANS, Rao $\&$ Venugopal (1985) give $0.12<E(B-V)<0.20$. From the interstellar lines present in the IUE spectrum, Parthasarathy et al. (1986) estimate $0.0<E(B-V)<0.15$. Later, the value $E(B-V)=0.20 \pm 0.05$ is adopted by Dudley \& Jeffery (1993) for determining of the $T_{\text {eff }}$ of the HdB star $v$ Sgr.

Assuming $R_{\mathrm{V}}=3.1$, this color excess gives a total visual absorption $A_{\mathrm{V}}=0.620 \pm 0.155$. This value does not contradict the value $A_{\mathrm{V}}=0.455_{-0.031}^{+0.034}$ computed with the analytic expression proposed for the interstellar visual absorption by Chen et al. (1998) for the galactic latitude $b=-13.8^{\circ}$ and the revised distance from Hipparcos.

Considering these low values for the estimated interstellar absorption and the large infrared excess exhibited by the source, it naturally comes out that the circumstellar dust around $v \mathrm{Sgr}$ should exhibit a small self-absorption. Given the asymmetry of the source, the dust cannot be distributed in a spherical shell but rather by a disk that does not intercept the line-of-sight and must be seen at low or intermediate inclination.

\subsection{The SED of $v$ Sgr}

The SED of $v$ Sgr was derived from UV, visual, near-IR, and mid-IR measured flux $f(\lambda)$ (in erg/s $/ \mathrm{cm}^{2} / \mathrm{Hz}$ ) compiled by Trams et al. (1991). The dereddened flux $f_{0}(\lambda)$ is computed using the relation:

$f_{0}(\lambda)=f(\lambda) 10^{0.4 R(\lambda) E(B-V)}$.

The value of $R(\lambda)$ was taken from the analytic expression of the interstellar law from Cardelli et al. (1989) with a ratio of total to selective extinction $R_{\mathrm{V}}=3.10$. For $v$ Sgr we adopted the value of the color excess $E(B-V)=0.20$ from Dudley \& Jeffery (1993). This flux was converted into SI units via $F_{0}(\lambda)\left(\mathrm{W} / \mathrm{m}^{2} / \mu \mathrm{m}\right)=$ $3.0 \times 10^{-11} f_{0}(\lambda) / \lambda(\mu \mathrm{m})$.

The resulting SED is shown in the Fig. 2. The SED of $v$ Sgr clearly appears to be composite, and it can be fitted by two blackbodies. One represents the radiation of the stellar system at $T=12000 \mathrm{~K}$, the other the radiation from the dusty envelope at $T=950 \mathrm{~K}$ seen in the mid-IR. We derived the luminosity of the central source $L=39000_{-9100}^{+13300} L_{\odot}$ using the Hipparcos distance $d=595_{-72}^{+94} \mathrm{pc}$ and by fitting the dereddened SED with 2 black-body spectra as seen in Fig. 2.

As mentioned in the previous section, we can expect some silicates in the dusty envelope of the binary, as suggested by the $10 \mu \mathrm{m}$ feature in the MIDI spectra. It might be inconsistent however, to restrict the chemical composition to the silicate dust, as the primary source is now a very hydrogen-deficient star (for the possible evolutionary scenario see Delgado \& Thomas 1981).

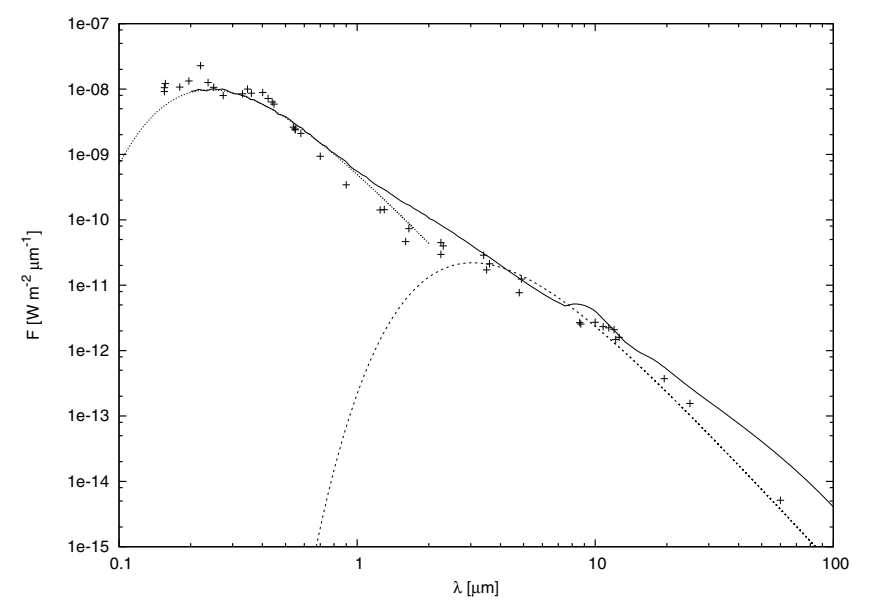

Fig. 2. Spectral energy distribution of $v$ Sgr from Trams et al. (1991). The dashed lines are the blackbody fits with temperatures $12000 \mathrm{~K}$ and $950 \mathrm{~K}$. The solid line is the model SED for the best visibilities model.

\subsection{MC3D description}

We used the MC3D code from Wolf et al. (1999) to interpret the interferometric data and the observed SED. MC3D is a self-consistent continuum radiative transfer code based on the Monte-Carlo method for emitting, scattering, absorbing, and reemitting the photons. The code assumes a spherical source located in the center of the coordinate system and spherical dust grains.

The density of the dust shell is computed according to the built-in geometry and input parameters. We used the model of the stratified dusty disk (Shakura \& Syunyaev 1973; Wood et al. 2002; Chesneau et al. 2006), the density of which is defined with the 2D law

$\varrho(r, z)=\varrho_{100}\left(\frac{100}{r}\right)^{\alpha} \exp \left[-\frac{1}{2}\left(\frac{z}{h(r)}\right)^{2}\right]$

where $r$ is the radial distance in the midplane of the disk, $\alpha$ the density parameter in the midplane, and the disk scale height $h(r)$ is given by $h(r)=h_{100}\left(\frac{r}{100}\right)^{\beta}$, where $\beta$ is the vertical density parameter and $h_{100}$ is the scale height at the distance of $r=100 \mathrm{AU}$. The source is described with two parameters: the effective temperature $T_{\text {eff }}$ and the luminosity $L$.

The dust is characterized by its chemical composition and the size of the grains. We used the astronomical silicate and amorphous carbon dust catalogs and tested several mixtures with different ratio of these two components. $v$ Sgr is an evolved object probably exhibiting double dust composition. A silicate feature can be seen in the SED Figs. 2 and 3. We assumed the grain size distribution of (Mathis et al. 1977) $\frac{\mathrm{d} n(a)}{\mathrm{d} a} \sim a^{-3.5}$, where $a$ is the dust grain radius. The grain radii were assumed to range from 0.01 to $1.00 \mu \mathrm{m}$.

\subsection{Visibility and SED model}

Because the current knowledge about the geometry of the binary orbit and its surrounding is poor, the parameter space of the models we had to explore was large; consequently, we were not able to explore the whole parameter space. The only fixed parameters were the parameters of the central source $\left(T_{\text {eff }}=12000 \mathrm{~K}\right.$, $L=39000 L_{\odot}$ ), which were derived from the fit of the dereddened SED as described in Sect. 3.2. Because we used several dust catalogs with different mixtures of silicates and carbon, we 


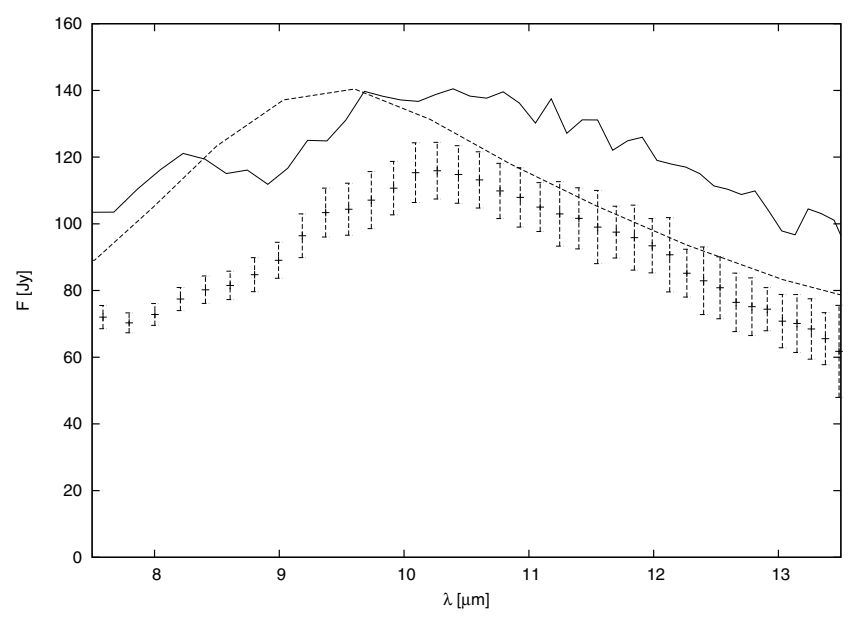

Fig. 3. Spectrum of $v$ Sgr taken with VLT/UTs (points with errorbars), the IRAS LRS (solid line), and SED of the best visibility fit model (dashed line). The $30 \%$ of extra flux of the IRAS LRS comes from the wider FOV of the IRAS spectrograph.

Table 3. Parameters of the model of the best fit of the visibility data and estimated uncertainties. Some parameters are poorly constrained and lack an error bar (see Sect. 3.4 for further details).

\begin{tabular}{cc}
\hline \hline Parameter & Value \\
\hline$R_{\text {in }}[\mathrm{AU}]$ & $6.0_{-1.5}^{+0.5}$ \\
$i$ & $50_{-20^{\circ}}^{+10^{\circ}}$ \\
$\alpha$ & $2.0_{-0.3}^{+0.3}$ \\
$\beta$ & 0.7 \\
$h_{100}[\mathrm{AU}]$ & $3.5_{-1.5}^{+2.0}$ \\
$\log \left(M_{\mathrm{d}} / M_{\odot}\right)$ & -3.5 \\
$M_{\text {am.c }} / M_{\mathrm{d}}$ & $0.6_{-0.4}^{+0.2}$ \\
\hline
\end{tabular}

adopted the sublimation limit of $1500 \mathrm{~K}$ for all the models. We also kept the outer radius of the model grid to $R_{\text {out }}=100 \mathrm{AU}$, which corresponds to the observed diameter of 400 mas. This is fully consistent with the image taken with VLT UT telescope, whose shows a perfect Airy pattern, which FWHM is approximately 225 mas at $8.7 \mu \mathrm{m}$.

The geometry of the disk is defined by $\alpha, \beta, h_{100}, R_{\text {in }}$, the inclination $i$, together with the total mass $M_{\mathrm{d}}$ of the dust and its composition. The fitting proceeded in two steps: in the first step, we focused on finding the best brightness distribution on the sky, i.e. concentrating on visibilities alone, which are influenced by the geometry of the dusty envelope. In the second step, based on the best models fitting the visibilities, we tried to find the best fit of the SED, taking particular care to reproduce the $10 \mu \mathrm{m}$ silicate feature.

The evaluation of the quality of the visibility fit was made by using the reduced $\chi^{2}$ calculated with the standard equation:

$\chi^{2}=\frac{1}{n} \sum_{i=1}^{n} \frac{\left(V_{\mathrm{obs}, i}-V_{\mathrm{model}, i}\right)^{2}}{\sigma_{i}^{2}}$,

where $V_{\mathrm{obs}, i}$ is the observed visibility, $V_{\text {model }, i}$ the model visibility interpolated to the same wavelength as the one of the observed visibility, and $\sigma_{i}$ the estimated error of the visibility. The parameters of the best model are shown in Table 3. The corresponding SED is shown in Figs. 2 and 3. The image of the disk given by the best model is shown in Fig. 6. The fit of the observed visibilities is shown in Fig. 5.

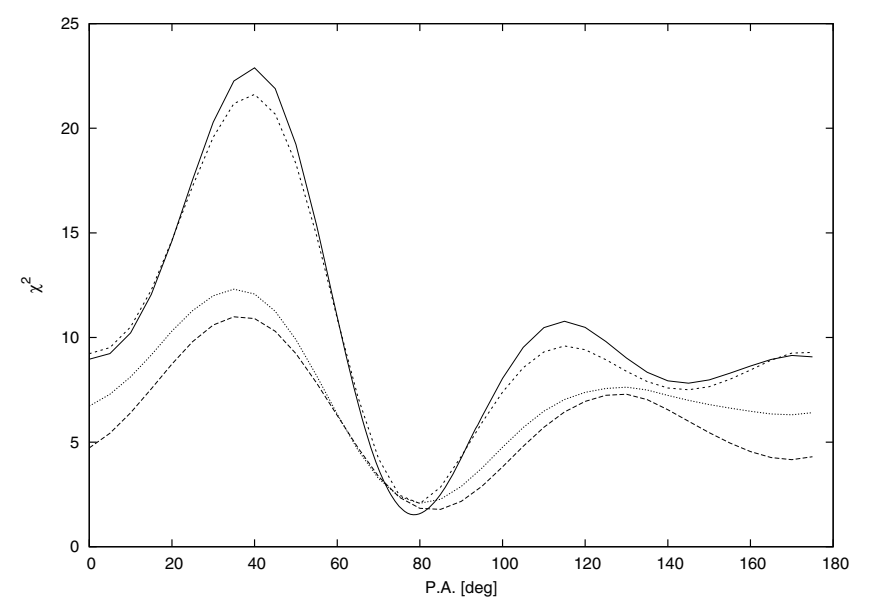

Fig. 4. The $\chi^{2}$ as a function of PA for several models that provide best visibility fits.

From the analysis of the computed models we can put the following constraints on the parameters of the model:

- $R_{\text {in }}$ : the inner radius of the dusty envelope is one of the most sensitive parameters. We find that, by using the visibilities fit, $R_{\text {in }}=6.0_{-1.5}^{+0.5} \mathrm{AU}$. This value is fully consistent with the estimated size of the binary inside the disk, whose upper limit for the semi-major axis is $1.9 \mathrm{AU}$ as mentioned in Sect. 1. The other constraint on the inner radius comes from the temperature distribution, close to the lower limit of $R_{\text {in }}$, the amount of the dust, which is above the sublimation limit temperature, is rapidly growing. Taking this into account, we conclude that the inner rim of the dusty disk is defined by its sublimation radius rather than by the binary orbit. However, the binarity of the source inside the disk can influence the disk morphology, but our models cannot include this feature.

- $\alpha, \beta, h_{0}$ : the constraints on the morphology of the object are not so strong. The $\alpha$ parameter is not defined very well and it provides satisfactory fits for reasonable values typically ranging from 1.8 to 2.4. Similarly, the low values of $\beta$ provide equally good fits, although we can firmly exclude any model with $\beta>1.0$, which means that the dusty disk of $v$ Sgr does not flare much. This can be indirect evidence of a Keplerian disk. This can also be seen as evidence of a self-shadowed puffed-rim disk (for discussion of the effects of different geometries see Dullemond \& Dominik 2004), a geometry not handled by our version of the MC3D code. Indeed, such geometry strongly enhances the near-IR flux of the SED, a part not well-fitted by the model. Our simulations give the limits for the scale height $h_{100}=3.5_{-1.5}^{+2.0} \mathrm{AU}$.

- $i$ : the inclination of the dusty envelope is one of the most interesting parameters of our model. If we assume that the dust envelope lies in the same plane as the binary, we put some constraints on the mass of the binary system. We find an inclination $i=50_{-20^{\circ}}^{\circ}$, which is consistent with the constraints mentioned in Koubský et al. (2006). Because the height scale $h_{100}$ is not defined well and because it is very difficult to distinguish between the flux coming from the inner rim of the disk and the "upper" surface in the mid-IR, since the disk is optically thin, it would be very hard to determine the inclination of the dusty envelope better, even with a uv space that is more densely covered.

- PA: as seen in Fig. 4, there is a clear minimum of $\chi^{2}$ for the PA of $80^{\circ}{ }_{-5^{\circ}}^{\circ}$. This orientation is almost perpendicular 

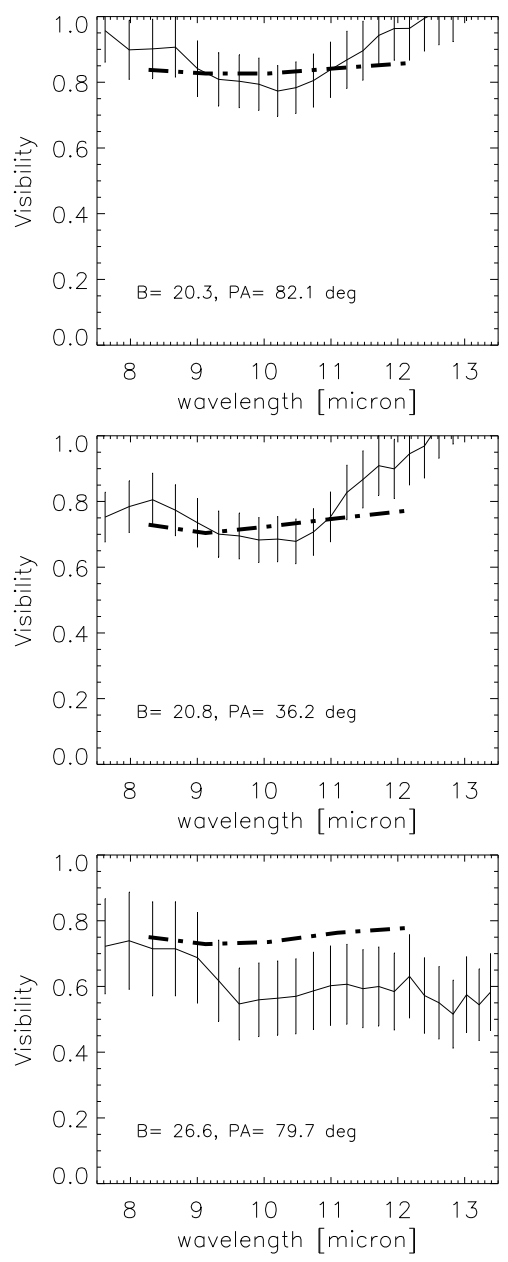
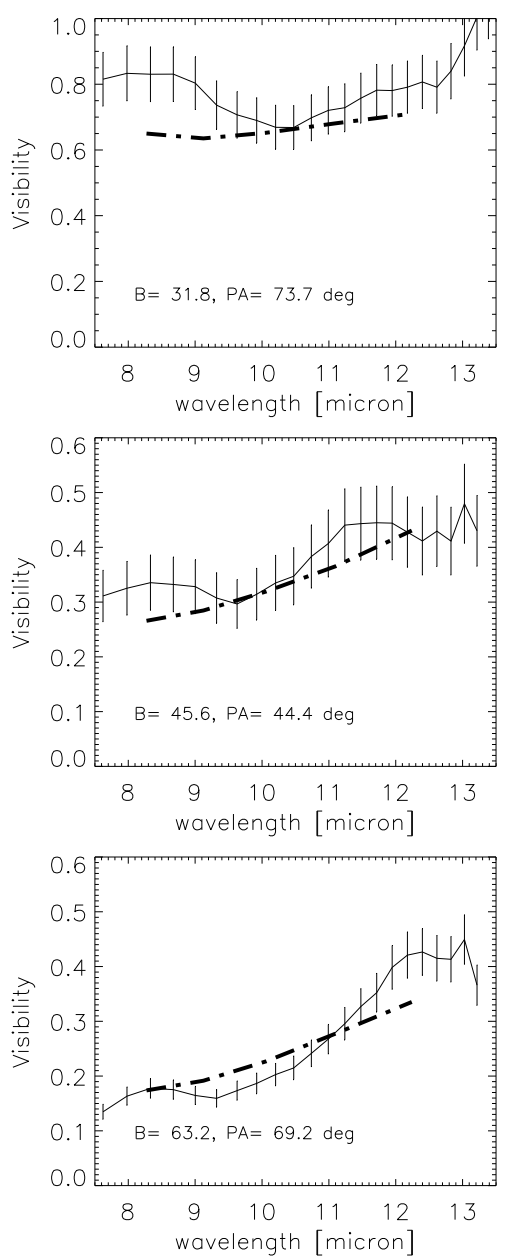
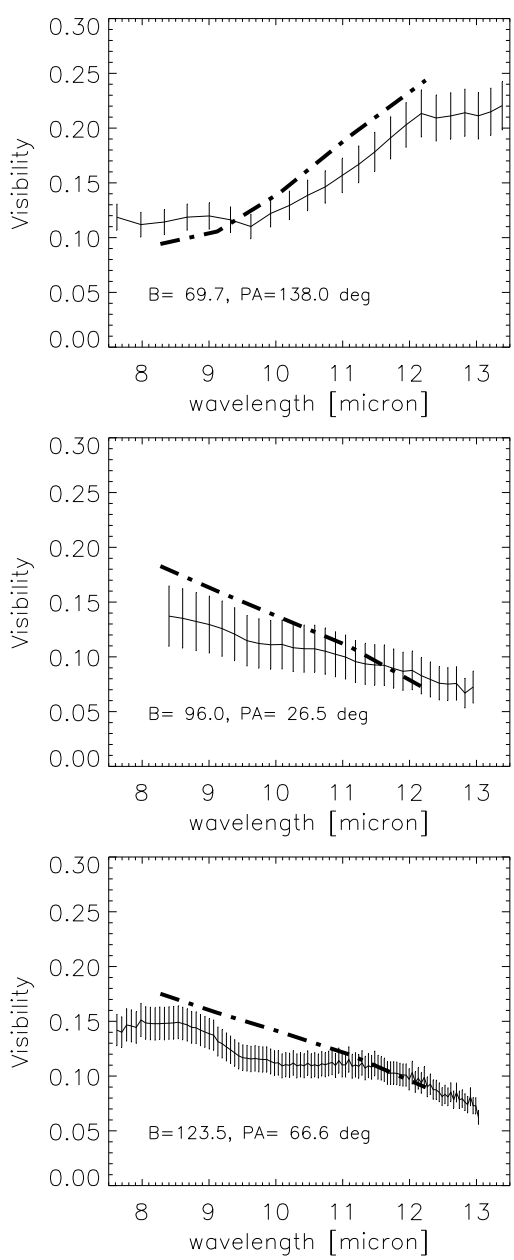

Fig. 5. The best visibility fit found $\left(\chi^{2}=1.5\right)$. The parameters of the model are the reference parameters in Table 3 . Note the different ranges on the visibility axes.

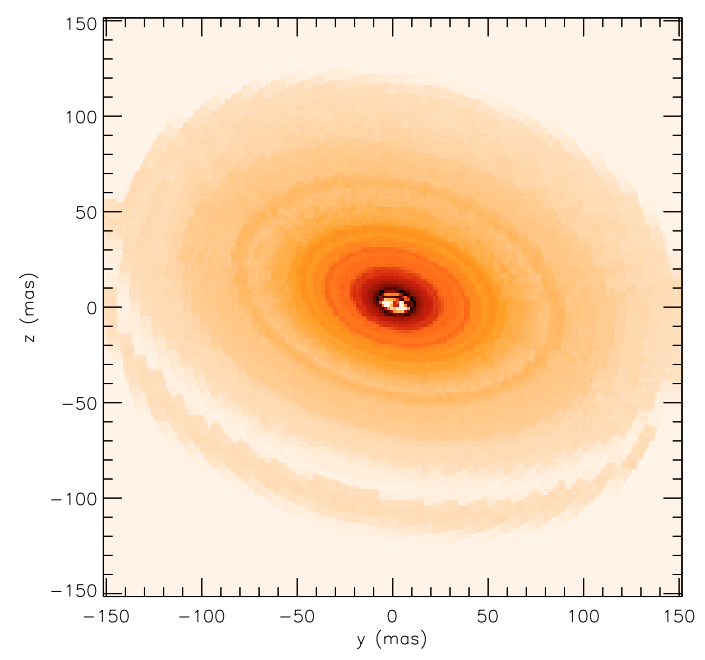

Fig. 6. The intensity map at $10.6 \mu \mathrm{m}$ for the best model of the visibilities. The intensity levels of the image corresponds to the $I^{\frac{1}{2}}$ of the real image.

to the direction of the linear polarization $P_{\mathrm{obs}}=172^{\circ}$ (data from Yudin 2001).

- $M_{\mathrm{d}}$, chemical composition: We have found only a weak dependency of the visibilities fits on the total mass of the dust. We explored a mass ranging from $5 \times 10^{-8}$ to $5 \times 10^{-3} M_{\odot}$ and the quality of the visibility fits, i.e. the overall appearance of the disk, changes only slightly. However, the chemical composition seems to be well defined, and the best results were obtained with dust mixture with $60 \%$ of carbon dust and $40 \%$ of silicate dust.

\section{Discussion}

\subsection{Limits of the model}

The model accounts for the main features of the observed SED (Fig. 2), i.e. the star and the dust emission, and the level of the silicate feature (Fig. 3). The main departure between the model and the observed SED is visible in the $\sim 1 \mu \mathrm{m}$ region. This is not surprising given the simplifications we imposed on these models. First, the binary source is simulated by a single, unresolved black-body in the center of the system. Second, no gas is included in the region between the central source and the sublimation radius. The existence of a disk of plasma is indirectly witnessed by the emission lines seen in the visible spectra (see Koubský et al. 2006). Such a disk may imply a strong source opacity viewed from the disk. Third, we crudely fitted the SED by changing the ratio of the silicates versus the carbon dust. The carbon dust is essentially a continuum, and the silicate peak from the model is broader than in the MIDI spectrum. It is not sure that the continuum and the $10 \mu \mathrm{m}$ feature stew from such dust compounds. A double chemistry is suspected for this source, but in 
the absence of clear carbon signature, such as the ones exhibited by PAHs, it is not obvious to define the dust content. It is, however, probable that the disk around the binary star passed through several periods of the dust settling: first with the oxygen-rich dust that formed the silicates dust, second with the carbon-rich dust, in agreement with the evolutionary scenario proposed by Delgado \& Thomas (1981). Moreover, as already pointed out, the geometric description of the inner rim is very simple.

\subsection{Consequences for the system orbital parameters}

These observations allowed us to restrict the interval of confidence for the inclination and position angle of the system. Using the new parameters and the updated Hipparcos parallax, it is now clear that $v$ Sgr harbors two massive stars. Referring to Table 5 in Koubský et al. (2006), the current mass of the two stars is higher than 10 solar masses. In this context, given the temperature and the mass of the components, it is not excluded that a kind of wind-wind collision in the system favors the formation of dust in the interface.

We demonstrated that a model involving a passive, stratified dusty disk can account for the rough properties of the infrared SED and the MIDI visibilities. It is useful to examine the residuals of the fits, as they contain some information on the departure of the flux distribution from the model compared to the observations. In particular, an imprint of the orbital motion on the dust formation, hence on the disk appearance, might be detectable. After examining the residual, however, we find that their shape is often similar, regardless of the projected baseline. This is due to the uncertainties on the dust composition, which dominantly affect the $10 \mu \mathrm{m}$ feature, giving rise to a permanent spectrally dependent residual. Nevertheless, the two visibility curves acquired with the UTs, reaching $100 \mathrm{~m}$, are less sensitive to these mismatches and more sensitive to compact structures that are not accounted for by the model. Noteworthy is that a small amplitude sinusoidal pattern is visible in the visibility curve of the longest baseline $\left(B \sim 123 \mathrm{~m}, \mathrm{PA}=66.6^{\circ}\right)$, almost aligned to the major axis of the disk, while such a pattern is clearly absent in the other $\left(B \sim 96 \mathrm{~m}, \mathrm{PA}=26.5^{\circ}\right)$, and is much smoother. This may suggest some low-contrast dusty structures, such as clumps (flux limited to $0.5-1 \mathrm{Jy}$ ). Given the binary nature of $v$ Sgr and the large amount of the dust around the system, the spiral pattern (a "pinwheel" nebula), as proposed by Nariai (1967) to account for the shifts in the $\mathrm{H} \alpha$ emission, may also be seen in the mid-IR. If such a pattern exists, it would be more favorably detected using near-IR interferometry with small baselines, or aperture-masking with large telescopes.

\section{Summary and conclusion}

The MIDI observations provide evidence of a thin, flat circumbinary disk around the hydrogen-deficient binary $v$ Sgr, whose inner rim lies close to the radius of sublimation temperature. The parameters of the disk provide better constraints on the PA and on the inclination of the orbital plane, allowing us to set the mass of the components to be higher than the typical $10 M_{\odot}$. However, there are still many open issues: e.g. the explanation of the peculiar spectrum of the "invisible" component and the verification of the radial velocity curve of the secondary, which can really be tested only at shorter wavelengths. The disk's inner rim can be studied best in the near-IR using short baselines $(\leq 40 \mathrm{~m})$. The components of the system, with a semi-major axis estimated to range between 2 mas and 4 mas, can be resolved with an interferometer operating in the near infrared (with baselines longer than $80 \mathrm{~m}$ ) or, better, with short baselines in the visible (continuum and some chosen lines) using the VEGA recombiner of the CHARA interferometer (Mourard et al. 2008).

Acknowledgements. We would like thank to A. Spang for his help on the data handling. This research made use of the Simbad database, operated at the Centre de Données Astronomiques de Strasbourg (CDS), France. We profited from electronic bibliography maintained by the NASA/ADS system. The preparation of the interferometric observations was made with ASPRO (the interferometric observing preparation software developed by the Jean-Marie Mariotti Center (JMMC, http: //mariotti.fr, Duvert et al., SPIE 4844, 60, 2002). The research of P. Harmanec was supported by grants 205/06/0304 and 205/08/H005 of the Czech Science Foundation and also by the Research Program MSM0021620860 Physical study of objects and processes in the solar system and in astrophysics of the Ministry of Education of the Czech Republic. The visit of M. Netolický to the Observatoire de la Côte d'Azur, where he was trained in modern VLTI software techniques and their application, was funded from the OPTICON grant R113-CT-2004-001566 JRA 4.

\section{References}

Ak, H., Chadima, P., Harmanec, P., et al. 2007, A\&A, 463, 233 Batten, A. H. 1970, PASP, 82, 574

Campbell, W. W. 1899, ApJ, 10, 241

Cardelli, J. A., Clayton, G. C., \& Mathis, J. S. 1989, ApJ, 345, 245

Chen, B., Vergely, J. L., Valette, B., \& Carraro, G. 1998, A\&A, 336, 137

Chesneau, O., Collioud, A., De Marco, O., et al. 2006, A\&A, 455, 1009

Delgado, A. J., \& Thomas, H.-C. 1981, A\&A, 96, 142

Dudley, R. E., \& Jeffery, C. S. 1990, MNRAS, 247, 400

Dudley, R. E., \& Jeffery, C. S. 1993, MNRAS, 262, 945

Dullemond, C. P., \& Dominik, C. 2004, A\&A, 417, 159

Duvignau, H., Friedjung, M., \& Hack, M. 1979, A\&A, 71, 310

Eggleton, P. P. 2002, ApJ, 575, 1037

Frame, D. J., Cottrell, P. L., Gilmore, A. C., Kilmartin, P. M., \& Lawson, W. A. 1995, MNRAS, 276, 383

Gehrz, R. D., Hackwell, J. A., \& Jones, T. W. 1974, ApJ, 191, 675

Hack, M., \& Pasinetti, L. 1963, Contr. Oss. Astr. Milano-Merate, 215, 1

Harmanec, P. 2002, Astron. Nachr., 323, 87

Harmanec, P., Morand, F., Bonneau, D., et al. 1996, A\&A, 312, 879

Hoffman, J. L., Nordsieck, K. H., \& Fox, G. K. 1998, AJ, 115, 1576

Koubský, P., Harmanec, P., Yang, S., et al. 2006, A\&A, 459, 849

Kuiper, G. P. 1941, ApJ, 93, 133

Leinert, C., Graser, U., Przygodda, F., et al. 2003, ApSS, 286, 73

Leinert, C., van Boekel, R., Waters, L. B. F. M., et al. 2004, A\&A, 423, 537

Mathis, J. S., Rumpl, W., \& Nordsieck, K. H. 1977, ApJ, 217, 425

Mourard, D., Perraut, K., Bonneau, D., et al. 2008, Proc. SPIE, 7013, 701323

Nariai, K. 1967, PASJ, 19, 564

Parthasarathy, M., Cornachin, M., \& Hack, M. 1986, A\&A, 166, 237

Perryman, M. A. C., Lindegren, L., Kovalevsky, J., et al. 1997, A\&A, 323, L49

Rao, N. K., \& Venugopal, V. R. 1985, J. Astrophys. Astron., 6, 101

Schmitt, H. R. 2008 [arXiv: 0801.4772]

Schoenberner, D., \& Drilling, J. S. 1983, ApJ, 268, 225

Schoenberner, D., \& Drilling, J. S. 1984, ApJ, 276, 229

Shakura, N. I., \& Syunyaev, R. A. 1973, A\&A, 24, 337

Shallis, M. J., \& Blackwell, D. E. 1979, A\&A, 79, 48

Trams, N. R., Waters, L. B. F. M., Lamers, H. J. G. L. M., et al. 1991, A\&AS, 87,361

Tuthill, P. G., Monnier, J. D., \& Danchi, W. C. 1999, Nature, 398, 487 van Leeuwen, F. 2007, A\&A, 474, 653

Walker, H. J. 1985, A\&A, 152, 58

Waters, L. B. F. M., Cami, J., de Jong, T., et al. 1998, Nature, 391, 868

Wilson, R. E. 1913, Lick Observatory Bulletin, 8, 132

Wolf, S., Henning, T., \& Stecklum, B. 1999, A\&A, 349, 839

Wood, K., Lada, C. J., Bjorkman, J. E., et al. 2002, ApJ, 567, 1183

Yudin, R. V. 2001, VizieR Online Data Catalog, 336, 80912

Zhao, M., Gies, D., Monnier, J. D., et al. 2008, ApJ, 684, L95 